\title{
Manganese doped cadmium sulphide (CdS: Mn) quantum particles: Topological, photoluminescence and magnetic studies
}

\author{
P. Venkatesu ${ }^{1 *}$, K. Ravichandran ${ }^{2}$ \\ ${ }^{I}$ Department of Physics, N. B. K.R Institutes, Vidyanagar 524413, India \\ ${ }^{2}$ Department of Nuclear Physics, University of Madras, Chennai 600025, India
}

*Corresponding author. E-mail: venkateshpudi@yahoo.co.in

Received: 23 July 2012, Revised: 24 August 2012 and Accepted: 26 August 2012

\section{ABSTRACT}

Nano-polycrystalline samples of cadmium sulphide doped with $0,2,4,6,8$ and 10 atomic \% of Mn were prepared by a template based chemical route. The presence of thiophenol as template on the surface of the samples has been detected with FTIR technique. Particle sizes of $15-50 \mathrm{~nm}$ range, hexagonal structure and polycrystalline nature of the samples have been identified through SEM and TEM techniques. Surface states in the band gap region of the samples confirmed by PL study also revealed that the size of the particles is in the nano range. The low temperature magnetization study suggested the 10 at.\% Mn doped sample might be in a re-entrant-spin glass phase at 77 K. Copyright $\odot 2013$ VBRI press.

Keywords: CdS: Mn; template; surface states; re-entrant-spin glass phase.

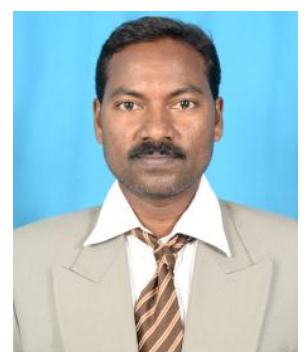

P. Venkatesu is currently working as a lecturer in the department of Physics, N.B.K.R Institutes, Vidyanagar, India. $\mathrm{He}$ obtained his graduate degree in Physics from Sri Venkateswara Arts College, Tirupathi, and M. Sc. from Sri Venkateswara University, Tirupathi in the year 1997. He did his Ph. D. in Physics in Diluted Magnetic Semiconducting nanomaterials at Sri Venkateswara University, Tirupathi in the year 2008. His research interests are Synthesis of Nanocrystalline Magnetic materials.

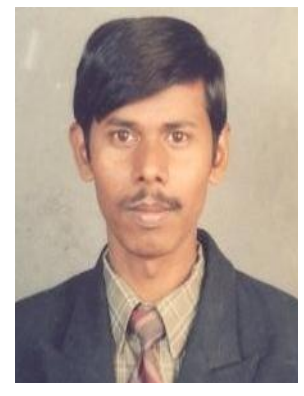

K. Ravichandran is a faculty in the Department of Nuclear Physics, University of Madras, Chennai India. He obtained his graduate degree in Physics from Madras University. M.Sc. and M. Phil received from University of Madras and Anna University, respectively. He obtained his $\mathrm{Ph}$. D. in Physics with Scientific Instrumentation from University of Madras Chennai. His Research interests are Synthesis of Nanocrystalline Magnetic materials, Metallic oxide materials, Spintronics and Nano-ferrofluids for drug delivery systems.

\section{Introduction}

The past few years have witnessed an exponential growth in the potential use of Diluted Magnetic Semiconducting (DMS) nanoparticles in diverse fields like material science, high density recording media, spin valves, magnetic resonance imaging, ferro fluid technology, magneto caloric refrigeration, environmental areas etc [1]. Recently, the researchers are highly interested in preparing multifunctional magnetic nanoparticles for biomedical applications such as target drug delivery, magnetic resonance imaging and magnetic hyperthermia treatment [2]. It was also reported that $\mathrm{CdS}$ doped with different transition metals could efficiently work as multifunctional nanoparticles. Zhang et al [3] reported white light emission in Eu, Dy co-doped $\mathrm{Y}_{2} \mathrm{Zr}_{2} \mathrm{O}_{7}$ crystalline powders. Sharma et al [4] reported that spherical nanoparticles of $\mathrm{Y}_{2} \mathrm{O}_{3}: \mathrm{Eu}^{3+}$ showed red luminescence, which could be used in flat panel display application. Sc/Y/Ti doped $\mathrm{CdO}$ has wide range of applications in optoelectronics was reported by Srivastava et al [5]. Yadav [6] prepared yttrium doped lead zirconium titanate with high ferroelectric and dielectric constant, has many potential applications in electronic devices. Radheshyam Rai [7] reported that $\mathrm{Zn}-\mathrm{Cu}$ doped $\mathrm{SnO}_{2}$ could be used as gas sensor instead of optical or electrochemical sensors to detect noxious gases in air. A novel saccharides detection assay based on covalent immobilization of amino phenyl boronic acid (APBA) in thin films of carboxyl functionalized chitosan containing $\mathrm{Gd}_{2} \mathrm{O}_{3}: \mathrm{Eu}^{3+}$ nanoparticles $(5 \mathrm{~nm})$ at a platinum disc electrode has been 
developed by Tiwari et al [8]. More recently, different types of nano materials compounds are being synthesized. In particular, star-shaped polymers in the nano-range might be useful for the controlled transport and release of several biologically active drugs [9]. On the other hand, Magnetic properties were also experimentally studied in some antiferromagnetic nanoparticles [10]. In this context, DMS nanoparticles are interesting materials due to their advantageous electronic and magnetic properties which are being widely studied for a better understanding on some fundamentally interesting issues related to their optical and magnetic properties of such wide band gap semiconducting materials [11]. Though extensive work reported on DMS, not much of work at the nano level of CdS:Mn specifically on the application to these properties has been attempted and hence our interest in this subject. In the present investigation the CdS:Mn nanoparticles having different 'Mn' content were prepared. Studies on composition, structure, morphology, photoluminescence and magnetic nature of the samples were carried out and interpreted.

\section{Experimental}

\section{Materials and apparatus}

Cadmium acetate (99+\%, Aldrich Chemical Co), manganese acetate $(99.1 \%$, Fisher Scientific) and $\mathrm{Na}_{2} \mathrm{~S} .9 \mathrm{H}_{2} \mathrm{O}$ (98\%, Aldrich Chemical Co) as precursors, methanol $\left(\mathrm{CH}_{3} \mathrm{OH}\right)$ as a solvent, thiophenol $\left(\mathrm{C}_{6} \mathrm{H}_{5} \mathrm{SH}\right)$ as a template and double distilled water obtained from commercial source( analytical reagent,AR grade) were used for the chemical co-precipitation synthesis of CdS: Mn nanocomposite system. A microbalance (M/s SICO, India) was used to weigh the materials to $10 \mu \mathrm{g}$ accuracy. Quartz tubes (M/s INFUSIL, India) were used to heat the samples in furnace which is run uninterruptedly using $10 \mathrm{KVA}(\mathrm{M} / \mathrm{s}$ Kirloskar, India) generator. It has provision of high vacuum system (M/s Avac, India).

\section{Preparation of CdS:Mn nanoparticles}

CdS:Mn powder samples were prepared by chemical route using their acetate salts. $2.6640 \mathrm{~g}$ of $\mathrm{Cd}\left(\mathrm{CH}_{3} \mathrm{COO}\right)_{2}$ was completely dissolved in $100 \mathrm{ml}$ of methanol to form $0.1 \mathrm{M}$ solution and $2.9964 \mathrm{~g}$ of $\mathrm{Mn}\left(\mathrm{CH}_{3} \mathrm{COO}\right)_{2}$ was completely dissolved in $100 \mathrm{ml}$ of methanol to form $0.1 \mathrm{M}$ solution. These two solutions were mixed in appropriate concentration to get the desired ratio of $\mathrm{Cd}: \mathrm{Mn}$ and the solution was taken in a burette. Similarly $0.1 \mathrm{M}$ solution of $\mathrm{Na}_{2} \mathrm{~S}$ with a few drops of thiophenol was prepared and this solution was taken in a conical flask. Thiophenol was used as both reductant and surfactant. Now the solution in the burette is added drop by drop into conical flask under continuous stirring for about 6 hours until fine precipitate of yellowish CdS: Mn was formed. Then the precipitate was filtered out separately and washed thoroughly with deionized water to remove the by-product, sodium acetate salt. Finally these samples were calcined at $300^{\circ} \mathrm{C}$ for $2 \mathrm{hrs}$ in vacuum to induce crystallinity, because the preliminary XRD studies on as prepared samples showed amorphous structure. A single step chemical reaction is given below for the precipitation of the Mn doped CdS nanoparticles

\section{$(1-\mathrm{x}) \mathrm{CdCH}_{3} \mathrm{COOH}+\mathrm{xMnCH}_{3} \mathrm{COOH}+\mathrm{Na}_{2} \mathrm{~S} \rightarrow$ $\mathrm{CdS}: \mathrm{Mn} \downarrow+2 \mathrm{NaCH}_{3} \mathrm{COOH}$}

\section{Characterization of CdS:Mn nanoparticles}

Scanning Electron Microscopy (SEM), with EDAX attachment camera (Model; JSM 840A) and scintillation counter detector, was used for both physical observations of the sample surfaces as well as chemical mapping. SIEFERT X-ray diffractometer (Model; 3003TT) was used for studying the structure of the samples. The morphologies of the products were investigated using a JEOL JEM- 3010 transmission electron microscopy (TEM). Fourier Transform Infrared Spectroscopy (FT-IR) spectrum of CdS:Mn nanoparticles was obtained using FT-IR spectrophotometer (Model; MIR $8300^{\mathrm{TM}}$ ) with $\mathrm{KBr}$ nanoparticles mixture in the pellet form. The fluorescent spectra of the samples were recorded using a fluorescence spectrophotometer (Hitachi F-3010). Magnetic properties of the samples were studied with vibrating sample magnetometer (VSM, Model; PAR 4500).

\section{Results and discussion}

In principle any method capable of producing very fine grain sized polycrystalline materials can be used to produce nanostructured materials. Chemical method is very cost effective and can yield fine grains [12]. Chemical coprecipitation method has been undertaken in the present investigation to prepare the samples of CdS:Mn. The interest in the Mn doped CdS is based on their outstanding magneto-optical properties caused by a strong $s, p-d$ exchange interaction between electron/hole band states and $\mathrm{Mn}^{2+} 3 d$ electron states. The samples of CdS:Mn were first subjected to chemical analysis using Energy Dispersive Analysis of X-rays (EDAX) method. The atomic percentages of the chemical species found in the samples are given in Table 1. The average deviation of the estimated compositions from the target compositions is within $\pm 2.5 \%$.

Table 1. EDAX compositional analysis of the samples.

\begin{tabular}{|c|c|c|c|c|c|}
\hline \multicolumn{3}{|c|}{$\begin{array}{l}\text { Starting elements in } \\
\text { at\% }\end{array}$} & \multicolumn{3}{|c|}{$\begin{array}{l}\text { Elements from EDAX analysis } \\
\text { in at } \%\end{array}$} \\
\hline $\mathrm{Mn}$ & $\mathrm{Cd}$ & $S$ & $\mathrm{Mn}$ & $\mathrm{Cd}$ & $S$ \\
\hline 0 & 50 & 50.0 & 0.00 & 47.23 & 52.77 \\
\hline 2 & 48 & 50.0 & 2.03 & 46.87 & 51.10 \\
\hline 4 & 46 & 50.0 & 4.09 & 43.85 & 52.06 \\
\hline 6 & 44 & 50.0 & 6.04 & 42.78 & 51.18 \\
\hline 8 & 42 & 50.0 & 8.07 & 42.61 & 49.32 \\
\hline 10 & 40 & 50.0 & 9.85 & 41.95 & 48.20 \\
\hline
\end{tabular}

XRD patterns of the samples are shown in Fig. 1. It is observed that there are well defined peaks corresponding to (100), (002), (101), (102), (110), (103) and (112) planes indexable to hexagonal phase of CdS [13]. A detailed study 
of the crystal structure of the CdS:Mn samples based on powder x-ray diffraction will be published separately [14].

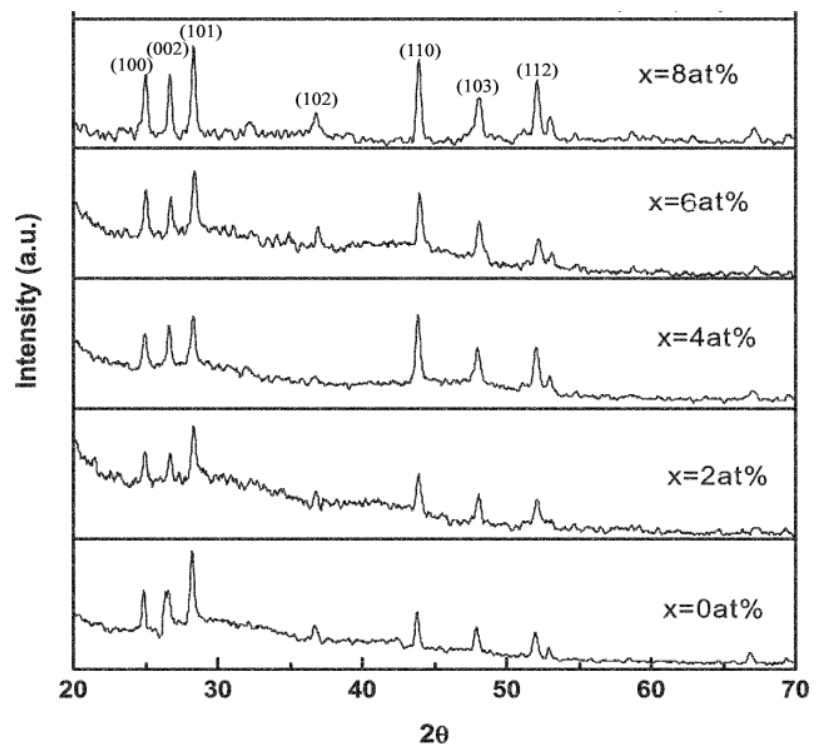

Fig. 1. XRDs of the CdS:Mn samples.

The morphology and topography of the samples were studied using imaging techniques such as Scanning Electron Microscopy (SEM) and Transmission Electron Microscopy (TEM). SEM image is shown in fig. 2, the rough and spongy surface morphology is evident which makes it difficult to estimate the crystallite size due to agglomeration of the particles.

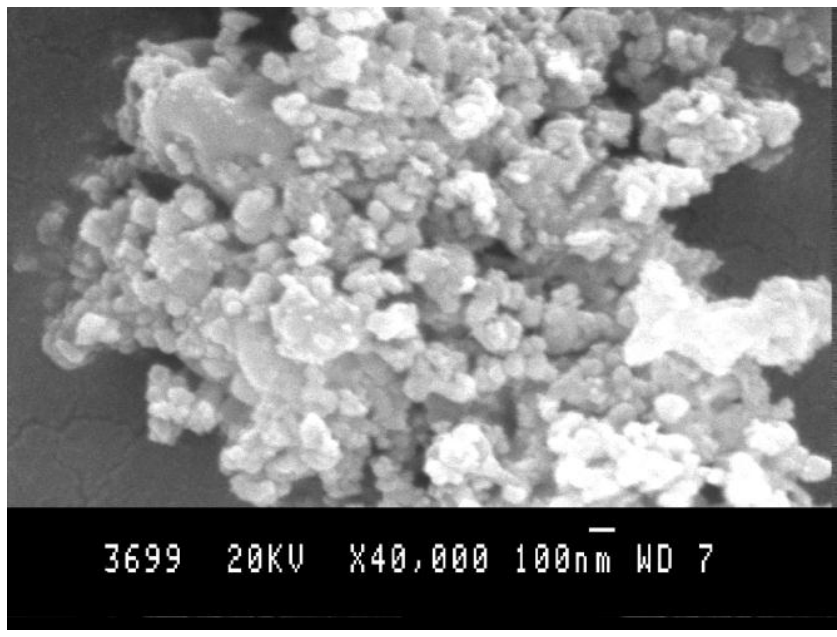

Fig. 2. SEM image of CdS:Mn nanoparticles.

TEM image is shown in Fig. 3 where (a), (b) and (c) are the bright field image, selected area electron diffraction (SAED) pattern and lattice fringes in the sample respectively. The size distribution in the bright-field image was measured by manual counting from a population of about 50 particles by skipping course particles. The particle size measured is about $15-50 \mathrm{~nm}$ range. Further, the quasispherical shape is also evident. In SAED pattern (101), (110) and (103) planes belong to wurtzite, hexagonal structure of CdS [15], are consistent with XRD results. The lattice fringe image of the sample discloses that the nanoparticles cores are polycrystalline in nature. Since, the orientation of atoms in different grains is different.
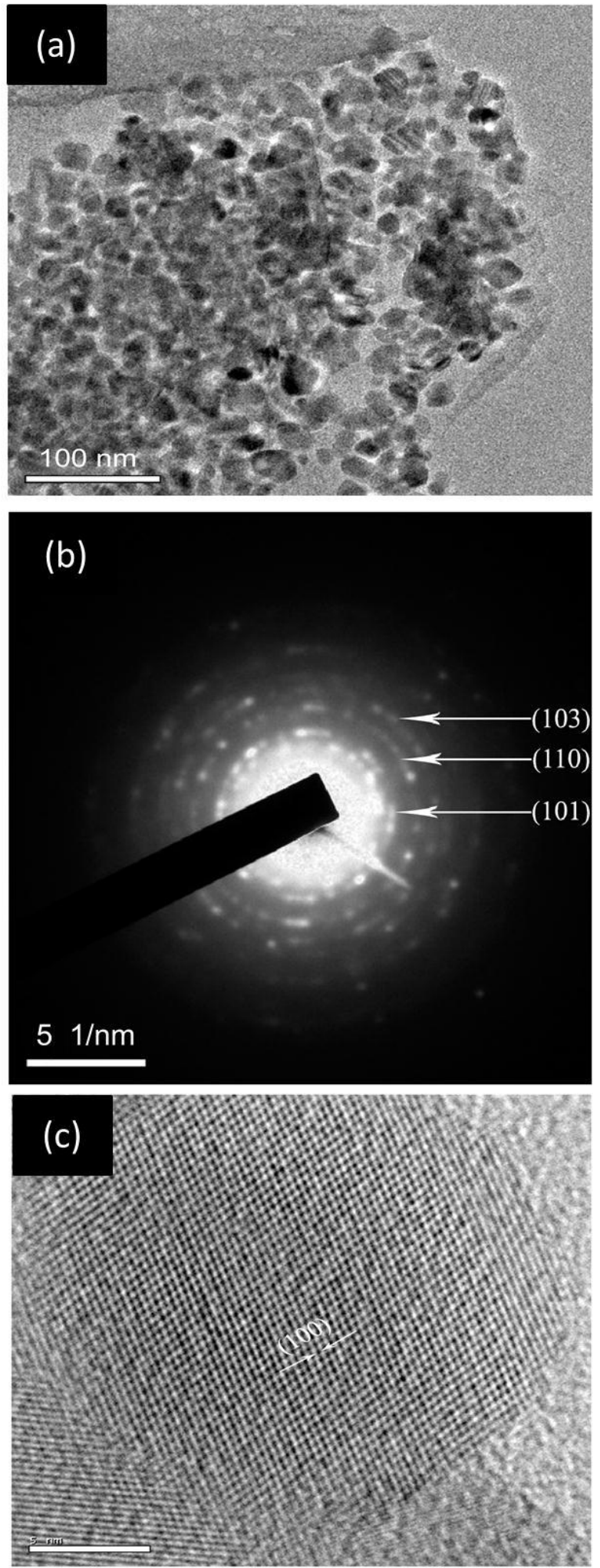

Fig. 3. HRTEM micrographs (a) Bright-field image (b) SAED pattern (c) Lattice fringe image.

FTIR spectrum is shown in Fig. 4. The peak at 3410 $\mathrm{cm}^{-1}$ is assigned to $\mathrm{O}-\mathrm{H}$ stretching of absorbed water on the surface of the sample and the peak at $1436 \mathrm{~cm}^{-1}$ is attributed to bending vibrations of methanol used in the 
process. The $\mathrm{C}-\mathrm{O}$ stretching vibration of absorbed methanol gives its intense peak at $1116 \mathrm{~cm}^{-1}$. In addition to surface coverage of $\mathrm{CdS}$ by methanol, presence of trace amounts of template ligand namely thiophenol is also evident, its ring $\mathrm{C}-\mathrm{H}$ vibration occurs at about $3000 \mathrm{~cm}^{-1}$ which is a very weak peak. Similar such weak peaks due to $\mathrm{C}-\mathrm{H}$ bending vibrations are observed at about $616 \mathrm{~cm}^{-1}$. Hence, presence of thiophenol in its dissociation form is obvious. Yang et al [16] also reported similar IR results with different template.

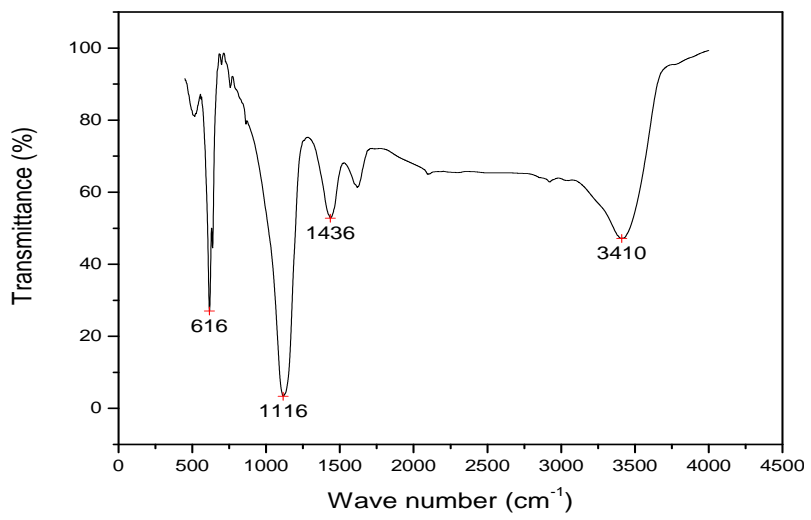

Fig. 4. FTIR spectrum of CdS:Mn sample.

Photoluminescence (PL) spectra of the samples are shown in Fig. 5. The excitation wavelength for all the samples is $350 \mathrm{~nm}$. The spectra exhibit a sharp emission band peaking around $380 \mathrm{~nm}$ for the samples of all compositions. In addition, there are also a series of emission bands between $440-500 \mathrm{~nm}$. The peak at $380 \mathrm{~nm}$ is attributed to the emission due to transition from the conduction band to the valence band of the CdS host [17]. This band gap emission is located in the energy region between the energy gap of bulk CdS $(2.52 \mathrm{eV})$ and that of $\mathrm{MnS}(3.5 \mathrm{eV})$, which is a combined result of the quantum size effect and the energy gap tuning effect for ternary compounds [18]. The series of emission bands are due to particles of different sizes. The appearance of the peaks at energies lower than the band gap [red shift], clearly demonstrates that the luminescence is from surface states [19]. The presence of these surface states reduces the chance of excitonic emission via non radiative surface recombination [20, 21]. The band edge or excitonic emission thus overlaps with the absorption of the surface states. This results in photoluminescence at energies less than the band gap energy.

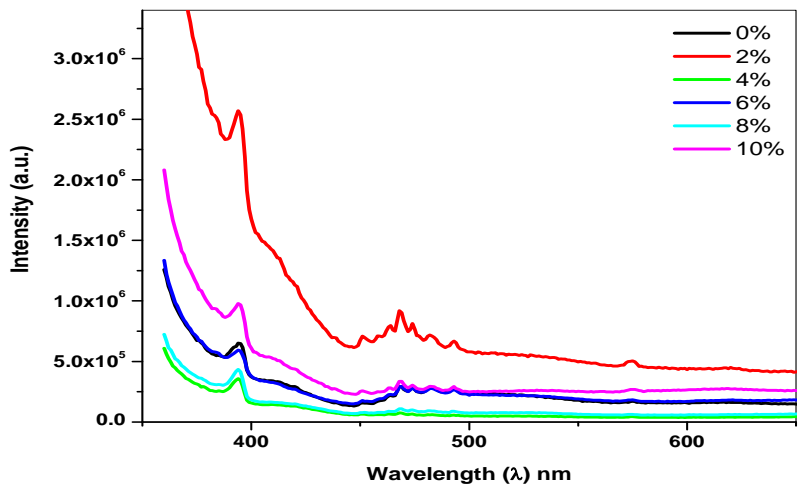

Fig. 5. PL spectra of CdS: Mn nanoparticles.
Magnetization measurements on $\mathrm{CdS}$ and Mn doped $\mathrm{CdS}$ samples were performed at room temperature in the range of applied magnetic field $0-10 \mathrm{kOe}$. The system was calibrated against a standard $\mathrm{Ni}$ sample with a known value of magnetic moment. The diamagnetic contribution from $\operatorname{CdS}\left(\chi_{\mathrm{Cd}}=-0.369 \times 10^{-6} \mathrm{emu} / \mathrm{g}\right)$ was then subtracted from the $\chi$ of doped samples [22]. Fig. 6 shows the variation of magnetization as a function of magnetic field for all the samples. The magnetization curves for the samples of CdS: Mn with Mn contents of $0,2,4,6$ and 8 at. $\%$ are all straight lines passing through the origin. No change in magnetization was observed when the field was reversed that is no significant hysteresis was noticed. This shows that the samples are in paramagnetic state and also co-operative magnetization effects are totally absent. But sample of 10 at. \% Mn exhibited small coercive field indicative of weak ferromagnetism and hence the sample presented a small hysteresis loop.

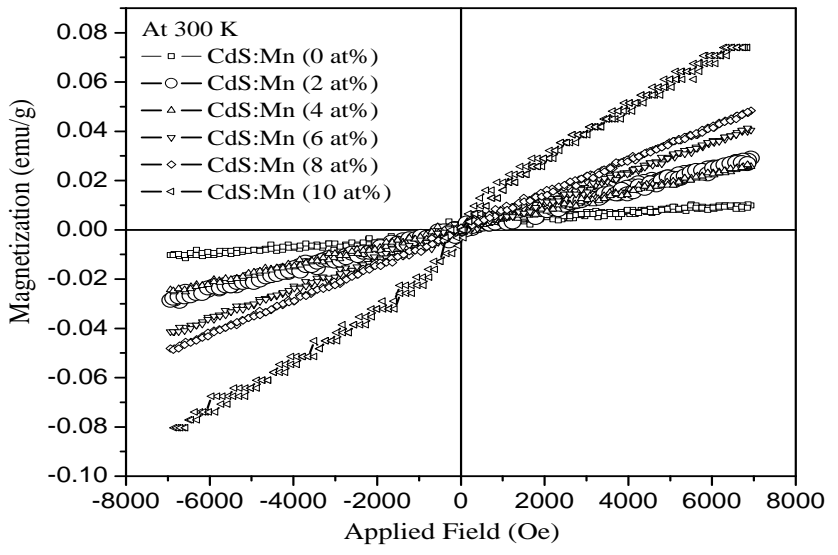

Fig. 6. $\mathrm{M}-\mathrm{H}$ hysteresis loops of the samples at $300 \mathrm{~K}$.

The nature of Paramagnetism in pure $\mathrm{CdS}$ might be attributed to super paramagnetic nature of the small grains present in the sample and also to spin canting /surface pinning effect. When the size of the particles is reduced below the single domain limit, they exhibit superparamagnetism at room temperature followed by a spin-glass like transition at low temperature [23]. Superparamagnetism is essentially a result of noninteracting, thermally fluctuating nanoparticles moments where the volume dependent effective anisotropy energy of each particle is easily overcome by the thermal energy $\left(\mathrm{K}_{\mathrm{B}} \mathrm{T}\right)$. The effect of this is that the nanoparticles have a large moment with high saturation magnetization (as expected in Ferro magnets) but a non hysteretic M-H curve with zero remanance and coercivity. The nature of magnetism in Mn doped CdS can be understood as follows. In these samples each $\mathrm{Mn}^{2+}$ has a total spin $\mathrm{S}=5 / 2$, originating from the half filled ' $3 \mathrm{~d}$ ' shells. $\mathrm{Mn}^{2+}$ spins are coupled by short-range anti-ferromagnetic interaction [24]. This interaction yields a series of steps at higher magnetic fields in the magnetization curves of $\mathrm{Mn}$ doped $\mathrm{CdS}$ nanoparticles. The phenomenon results in a progressive alignment of the nearest-nearest $\mathrm{Mn}$ pair spin components along the direction of magnetic field. The susceptibility varies non-linearly with concentration of $\mathrm{Mn}$ in $\mathrm{CdS}$. At low concentration of $\mathrm{Mn}$, the sp electron with opposite spin 
interacts with spin of $\mathrm{CdS}$ lattice and causes a decrease in susceptibility. At high concentrations of $\mathrm{Mn}$, the $\mathrm{d}-\mathrm{d}$ exchange interaction between $\mathrm{Mn}$ atoms dominates over the sp-d exchange interaction resulting in an abrupt increase in susceptibility.

In order to know the low temperature magnetic behavior of the Mn doped CdS samples, a typical M-H curve for 10 at. \% Mn doped CdS sample was taken at $77 \mathrm{~K}$. Normally for a DMS material at low temperature, a better signal indicating ferromagnetic ordering is expected. But the present measurements on 10 at. $\% \mathrm{Mn}$ at $77 \mathrm{~K}$ presented a distorted signal as shown in Fig. 7. There is no precise explanation for this observation, but this indicates the possibility of re-entrant spin-glass phase. In a re-entrant spin-glass phase, two phase transitions are observed; one is a ferromagnetic transition at a higher temperature and the second, a spin-glass transition at a lower temperature [25]. This actually shows the instability of the ferromagnetic phase at low temperatures. Magnetic measurement using SQUID magnetometer or other characterization aspects like magnetic circular dichroism (MCD) and magnetic optical Kerr effect (MOKE) may throw more light on this aspect.

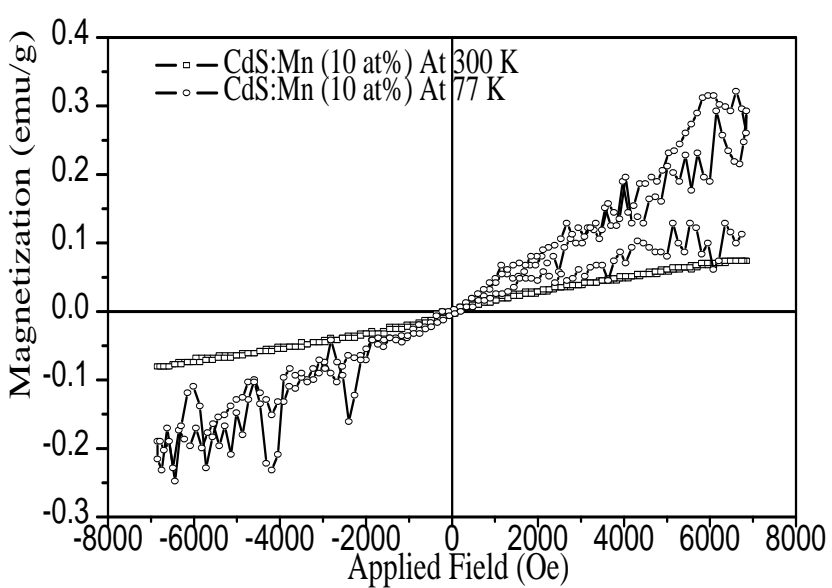

Fig. 7. M- H curve of 10at. \% Mn doped CdS at $77 \mathrm{~K}$.

\section{Conclusion}

Isolated $\mathrm{Ag}$ nanoparticles were synthesized on the surface by $150 \mathrm{keV}$ Ar ion irradiation of Ag-PVC bilayer films. Size of the Ag nanoparticles decreases with increasing ion fluence. The size distribution also decreases and becomes narrower with increasing ion fluence. Contact angle measurement of the surface before and after irradiation shows the surface roughness dependent and reveals that surface tends to hydrophilic with decreasing size of $\mathrm{Ag}$ nanoparticles and increasing ion fluence. Results are explained in view of sputtering and crater formation in $\mathrm{Ag}$ film and disorder created in polymer, through molten zones due to thermal spike induced by $150 \mathrm{keV}$ Ar ions.

\section{Reference}

1. Faraji, M.; Yamini, Y.; Rezaee, M. J. Iran. Chem. Soc. 2010, 37, 7. DOI: $\underline{10.1007 / \mathrm{BF} 03245856}$

2. Sharma, P.K.; Dutta, R.K.; Pandey, A.C. Adv. Mat. Lett. 2011, 2(4), 246. DOI: $10.5185 /$ amlett.2011.indias. 214

3. Zhang, A.; Yang, P.; Cao, Y.; Zhu, Y. Adv. Mat. Lett. 2011, 2(5), 322 .

DOI:10.5185/amlett.2011.3042am2011
4. Sharma, P.K.; Dutta, R.K.; Pandey, A.C. Adv. Mat. Lett. 2011, 2(4), 285 .

DOI: $10.5185 / a m l e t t$. Indias. 195

5. Srivastava, P.; Sharma, Y. Adv. Mat. Lett. 2011, 2(4), 290. DOI: $10.5185 /$ amlett.indias.206

6. Yadav, K.L. Adv. Mat. Lett. 2010, 1(3), 259. DOI: $10.5185 /$ amlett.2010.9159

7. Rai, R. Adv. Mat. Lett. 2010, $1(1), 55$ DOI:10.5185/amlett.2010.3101

8. Tiwari, A. et al, Anal. Methods, 2011, 3, 217. DOI:10.1039/COAY00574F

9. Tiwari, A. "Intelligent Nanomaterials", Wiley-Scrivener Publishing LLC, USA. ISBN: 978-04-709387-99, 2012.

10. Bodker, F.; Hansen, M.F.; Koch, C.B.; Morup, S. J. Magn. Magn. Mater. 2000b, 221, 32.

DOI: $10.1016 /$ so304-8853(00)00392-9

11. Benecke, C.; Busse, W.; Gumlich, H.E. J. Cryst. Growth 1990, 101, 931.

DOI: $10.1016 / 0022-0248(90) 91109-4$

12. Nosaka, Y.; Ohta, N.; Miyama, H. J. Phys. Chem. 1990, 94, 3752. DOI: $10.1021 / \mathrm{j} 100372 \mathrm{a} 073$

13. Xiong, Y.; Xie, Y.; Yang, J.; Zhang, R.; Wu, C.; Du, G. J. Mater. Chem. 2002, 12, 3714. DOI: $10.1039 / \mathrm{b} 206377 \mathrm{~h}$

14. Proceedings of the "National Conference on Trends in Renewable Energy, Sources, Applications and Technologies" organized by Centre for Nanoscience \& Nanotechnology, Sathyabama University in association with Ministry of Earth Sciences (MoES), New Delhi, 1-3 Feb. 2012. ISBN: 978-81-922119-3-0

15. Azad Malik, M.; Paul O \& apos; Brien, N.; Revaprasadu. J. Mater. Chem. 2001, 11, 2383. DOI: $10.1039 / \mathrm{b} 10270 \mathrm{n}$

16. Yang, J.; Zeng, J. H.; Yu, S. H.; Yang, L.; Zhou, G. E.; Qian, Y. T. Chem. Mater. 2000, 12, 3259.

DOI: $10.1021 / \mathrm{cm} 0000144$

17. Lippens, P.E.; Lannoo, M. Semicond. Sci. Technol. 1991, 6, A157A160. DOI: $10.1088 / 0268-1242 / 6 / 9 \mathrm{~A} / 030$

18. Goede, O.; Heinbrodt, W. Phys. Status Solidi 1988, B146, 11. DOI: $\underline{10.1002 / \mathrm{pssb} .2221460102}$

19. Eychmuller, A.; Hasserlbarth, A.; Katsikas, L.; Weller, H.; Ber. Eunseges. Phys. Chem. 1991, 95, 79. DOI: $10.1021 / \mathrm{j} 100154 \mathrm{a} 019$

20. Chen, W.; Wang, Z.; Lin, Z.; Lin, L. J.Appl. Phys. 1997, 82, 3111. DOI: $10.1063 / 1.366152$

21. Sekikawa, T.; Yao, H.; Hayashi, T.; Kobayashi, T. Solid State Commun. 1992, 83, 969. DOI: $10.1016 / 0038-1098(92) 90523-\mathrm{c}$

22. Chadi, D. J.; White, R. M.; Harrison, W. A. Phys. Rev. Lett. 1975, 35, 1372. DOI: $10.1103 /$ Phys Rev Lett.35.1372

23. Fiorani, D.; Lanotte, L.; Ronconi, F. J. Magn. Magn. Mater. 1992, 104, 141. DOI:10.1016/0304-8853(92)90738-A

24. Spalek, J.; Lewicki, A.; Tarnawski, Z.; Furdyna, J. K.; Galazka, R. R.; Obuszko, Z. Phys. Rev. 1986, B33, 3407. DOI: $10.1103 /$ PhysRevB.33.3407

25. Eggenkamp, P. T. J. et al. Phys. Rev. 1995, B51, 15250. DOI: $10.1016 / 0016-7037(95) 00353-3$ 